\title{
Comparação do nível de atividade física e força de preensão manual com o perfil bioquímico de doentes renais crônicos
}

\author{
Comparison of physical activity levels and handgrip with the biochemical profile \\ of chronic renal patients
}

Kelser de Souza Kocka, João Antônio Breda Neto ${ }^{b}$, Marcos de Oliveira Machado ${ }^{c}$

a Fisioterapeuta. Físico. Mestre em Ciências da Saúde. Professor dos Cursos de Fisioterapia e Medicina da Universidade do Sul de Santa Catarina (UNISUL). Tubarão, SC, Brasil.

b Médico. Graduado do Curso de Medicina da Universidade do Sul de Santa Catarina (UNISUL). Tubarão, SC, Brasil.

Farmacêutico. Bioquímico. Doutor em Farmácia Análises Clínicas; Coordenador do Laboratório de Pesquisa em Exercício e Saúde-LAPES da

Universidade do Sul de Santa Catarina (UNISUL). Tubarão, SC, Brasil

RESUMO

Objetivos: Comparar atividade física referida, força de preensão manual (FPM) e dados bioquímicos de nefropatas crônicos submetidos à hemodiálise (HD).

Materiais e Métodos: Foi realizado um estudo transversal através da coleta de dados bioquímicos, atividade física referida e FPM. Os dados foram inseridos no Excel ${ }^{\circledR}$ e analisados pelo SPSS 20.0. As variáveis foram comparadas entre sexo e dicotomizadas pela média da FPM (kgf) e nível de atividade física (METs-minutos/semana), analisando a diferença estatística por meio do teste de Mann-Whitney $(p<0,05)$.

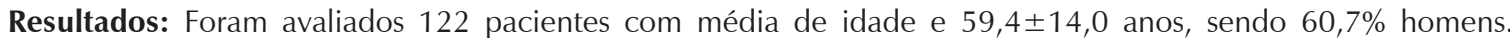
Comparando o sexo, os indivíduos do sexo masculino apresentaram maior FPM bem como níveis séricos de creatinina e hemoglobina, já os indivíduos do sexo feminino apresentaram melhores índices de depuração fracional da ureia (KT/V) e taxa de redução da ureia (URR). A FPM acima da média estratificada por sexo foi encontrada principalmente em pacientes mais jovens, que referiram praticar mais atividade física e possuíam maiores níveis séricos de albumina e creatinina. Nos indivíduos que possuíam moderado/alto nível de atividade física, foi observado maior FPM, maiores níveis de hemoglobina e menores valores de colesterol total.

Conclusão: Maiores níveis de atividade física e de FPM estão relacionadas à maior albumina e hemoglobina sérica e menor valor de colesterol total em pacientes renais crônicos.

Palavras-chave: insuficiência renal crônica; diálise renal; força da mão; dinamômetro de força muscular; exercício.

ABSTRACT

Objectives: To compare physical activity, handgrip strength (HGS) and laboratory profile in patients undergoing hemodialysis (HD). Materials e Methods: It was conducted a cross-sectional study through the collection of biochemical data, referred physical activity and HGS. Data were handled on Exce ${ }^{\circledR}$ and analyzed by the SPSS 20.0 program. The variables were compared between sex, dichotomized by the HGS average (kgf) and index of physical activity (METs-minutes/week). The statistical difference was analyzed using the Mann-Whitney test $(p<0.05)$.

Results: We evaluated 122 patients with a mean age of $59.4 \pm 14.0$ years, of which $60.7 \%$ were men. Comparing gender, males had higher HGS, serum creatinine and hemoglobin, while females had higher fractional clearance rates of urea (KT/V) and reduction rate of urea (URR). The HGS above average stratified by sex was found mainly in younger patients who reported more physical activity and had higher serum levels of albumin and creatinine. In patients with moderate/high level of physical activity, it was observed better HGS, as well as higher levels of hemoglobin and lower total cholesterol.

Conclusion: Higher levels of physical activity and higher HGS are related to greater serum albumin and hemoglobin and lower total cholesterol in patients at a chronic kidney condition.

Keywords: chronic renal insufficiency; renal dialysis; hand strength; muscle strength dynamometer; exercise. 


\section{INTRODUÇÃO}

A doença renal crônica (DRC) é definida como lesão renal com perda progressiva e irreversível da função dos rins e, em sua fase terminal, é denominada insuficiência renal crônica (IRC) $)^{1,2}$. Os estágios da DRC são classificados conforme a taxa de filtração glomerular (TFG) ${ }^{3}$. Sua estimativa pode ser obtida através da equação desenvolvida pelo grupo Chronic Kidney Disease Epidemiology Collaboration (CKD-EPI) que leva em conta: a idade (nos limites de 18 e 80 anos), o sexo, níveis séricos de creatinina e a etnia ${ }^{4}$. Os resultados obtidos a partir da equação podem ser distribuídos em cinco grupos. Quando há uma TFG inferior a $15 \mathrm{~mL} / \mathrm{min} / 1,73 \mathrm{~m}^{2}$, caracteriza-se a IRC. Na presença de IRC associada à uremia, indica-se a terapia renal substitutiva $(\mathrm{TRS})^{5}$.

A incidência média de pacientes submetidos à TRS no Brasil nos últimos 14 anos é de, aproximadamente, 4.121 pacientes/ano, sendo a grande maioria dos pacientes (84\%) custeados pelo Sistema Único de Saúde $(\mathrm{SUS})^{6}$. Das TRS, a hemodiálise (HD) é a mais difundida ${ }^{2,7}$ e a sobrevida dos pacientes submetidos à diálise crônica gira em torno de $75 \%$ ao final do quinto ano. Os principais fatores de risco de mortalidade são a idade, etnia caucasiana e presença de diabetes, não havendo diferença de sobrevida quando comparado sexo e tipo de diálise ${ }^{8,9}$. Pacientes que fazem HD geralmente apresentam anemia, fraqueza muscular, depressão, entre outros distúrbios que levam à redução progressiva da capacidade funcional e condicionamento físico ${ }^{2}$ que acarretam em um sentimento de invalide $z^{10}$.

Há também um comprometimento na capacidade cardiovascular, força e flexibilidade dos pacientes, culminando em dificuldades no desempenho de atividades diárias, de trabalho e lazer ${ }^{11}$. A redução de massa muscular é o mais importante marcador de mortalidade nos pacientes em HD. Esta redução dá origem a uma fraqueza generalizada que acarreta no "descondicionamento" físico, o qual está intimamente ligado a complicações cardiovasculares, a principal causa de morte durante o tratamento dialítico ${ }^{2}$. Como indicador de força e potência muscular uma das maneiras mais práticas e objetivas é a mensuração da força de preensão manual (FPM) aferida através de dinamômetro manual. A mesma é validada e utilizada como estratificação de risco em idosos ${ }^{12,13}$.

Embora haja necessidade de mais estudos para delinear as principais limitações físicas dos pacientes submetidos à diálise crônica, já se sabe que todos eles devem ser aconselhados a aumentar seu nível de atividade física, começando sempre de um nível baixo, conforme tolerado, em três ou mais dias da semana ${ }^{14,15}$. A quantidade de atividades físicas mínimas para um indivíduo deixar de ser sedentário varia de acordo com a referência utilizada, podendo ser avaliada através de questionários aplicados em forma de entrevista como o International Physical Activity Questionnaire (IPAQ), por exemplo ${ }^{16}$.

Também há evidências de que a FPM está associada á massa magra, estado nutricional e predição de complicações em pacientes renais crônicos ${ }^{17}$. Neste sentido, este trabalho visa comparar a relação dos parâmetros da bioquímica sanguínea com o índice de atividade física e a FPM de doentes renais. A hipótese deste estudo pondera que indivíduos com maiores níveis de atividade física e FPM possuam valores mais adequados aos níveis de referência da bioquímica sanguínea.

\section{MATERIAIS E MÉTODOS}

Foi realizado um estudo de delineamento transversal em 138 pacientes submetidos à terapia hemodialítica crônica, acompanhados entre o período de dezembro de 2014 a fevereiro de 2015. O trabalho foi autorizado pelo CEP em respeito à resolução 466 de 2012 do Conselho Nacional de Saúde, parecer número 732.861, CAAE: 32889714.6.0000.5369 na data de 24/07/2014 e pela Comissão de ética da instituição envolvida (Clínica de Doenças Renais, localizada em Tubarão-SC). Os dados foram coletados diretamente com os indivíduos submetidos à hemodiálise através de questionário e teste de força. Em um segundo momento, foram coletados dados do prontuário.

Foram incluídos no estudo indivíduos de ambos os sexos, acima de 18 anos que estavam em tratamento dialítico há pelo menos 01 (um) mês. Foram excluídos pacientes que apresentaram alterações dos sinais vitais durante a HD ou não tinham autonomia para decidir sobre sua participação no estudo. A recusa em participar não implicou de modo algum no atendimento oferecido no serviço de saúde ao paciente. $\mathrm{O}$ aceite para participar da pesquisa foi dado mediante anuência do termo de consentimento livre esclarecido (TCLE).

Foram utilizados 2 questionários, aplicados na forma de entrevista pelo pesquisador e um teste físico usando um dinamômetro para verificar a FPM. O primeiro questionário aplicado foi elaborado pelos pesquisadores contendo questões relacionadas à idade, sexo, peso, altura, índice de massa corpórea (IMC), escolaridade, história de tabagismo, tempo de hemodiálise em meses e presença ou ausência de outras comorbidades. Dados adicionais como valores plasmáticos de ureia e creatinina, albumina, depuração fracional da ureia (KT/V), taxa de redução da ureia (URR), hematócrito, hemoglobina, colesterol total e frações, triglicerídeos e hemoglobina foram coletados diretamente do prontuário. Também foram coletados do prontuário dados 
como peso, altura e tempo de hemodiálise em meses, para verificar com os dados fornecidos pelos pacientes. Esses dados, acrescidos do resultado do teste de força, ficaram anexados ao primeiro questionário. O segundo questionário foi o IPAQ em sua versão curta. Este questionário apresenta sete questões que permitem aferir o tempo semanal gasto em atividades físicas de intensidade leve, moderada e vigorosa $^{18}$. Para obtenção do nível de atividade física total em equivalentes metabólicos (MET)-minutos/semana foi utilizada a seguinte expressão: atividade física total MET-minutos/semana $=$ MET-caminhando + MET-moderado + MET-vigoroso. Onde MET-caminhando $=3,3 *$ minutos caminhando $*$ dias caminhando, MET-moderado $=4,0 *$ minutos de atividade de intensidade moderada* dias de atividades de intensidade moderada e MET vigoroso $=8,0 *$ minutos de atividade de intensidade vigorosa* dias de atividades de intensidade vigorosa ${ }^{19}$. Para a categorização do nível de atividade física foi considerado baixo quando os valores não ultrapassavam $600 \mathrm{METs-minutos/semana,} \mathrm{moderado}$ quando os valores estavam entre 600 e 3000 METs-minutos/ semana e alto quando estava acima de 3000 METs-minutos/ semana.

O instrumento utilizado para realizar a avaliação de FPM foi o dinamômetro hidráulico JAMAR ${ }^{\circledR}$ (Sammons Preston. Rolyan, 4, Sammons Court, Bolingbrook, IL, 60440, Canadá) que apresentou os resultados em quilograma força (kgf). O protocolo postural para a coleta de dados foi o proposto pela Sociedade Americana de Terapeutas de Mão (ASHT) ${ }^{20}$. Este protocolo recomenda que durante o procedimento os pacientes permaneçam sentados em uma cadeira tipo escritório (sem apoio para braços), com a coluna ereta. Também se deve manter o ombro posicionado em adução e rotação neutra com cotovelo flexionado a $90^{\circ}$ e antebraço em meia pronação com punho neutro. $\mathrm{O}$ punho pode ser movimentado em até $30^{\circ}$ graus de extensão e o braço deve ser mantido suspenso no ar com a mão posicionada no dinamômetro sendo este sustentado pelo examinador ${ }^{21,22}$. Em relação aos membros inferiores, foi permitido que os pacientes ficassem com o joelho em extensão, de maneira que a posição da extremidade inferior do corpo não interferisse no teste de força ${ }^{12}$. Esta decisão foi tomada para evitar instabilidades hemodinâmicas durante a HD. Nos pacientes que não possuíam fístula arterio-venosa (FAV), o membro de escolha para o teste foi o dominante. Nos casos em que o paciente possuía a FAV, o procedimento foi feito no braço contralateral do paciente. Tanto a coleta de dados quanto os testes citados foram feitos enquanto o paciente estava em diálise. Foram realizadas três medidas com tempo de contração isométrica de três segundos, com intervalo de descanso de um minuto. Apenas a FPM máxima foi anotada.
Os dados coletados foram inseridos no programa Excel ${ }^{\circledR}$ e a análise dos dados foi realizada no programa estatístico SPSS $20.0^{\circledR}$. As variáveis quantitativas foram descritas com medidas de tendência central e dispersão, e as variáveis categóricas foram descritas em números absolutos e proporções. As variáveis foram comparadas entre sexo, FPM $£$ média e FPM > média estratificadas separadamente por sexo, índice de atividade física baixo e moderado/alto, analisando a diferença estatística por meio do teste de MannWhitney $(p<0,05)$. Nas variáveis com diferença significativa foi analisado odds ratio (OR) por meio de regressão logística utilizando como variáveis dependentes a FPM dicotomizada e índice de atividade física baixo e moderado/alto.

\section{RESULTADOS}

Conforme metodologia proposta, 122 indivíduos foram avaliados, 8 recusaram-se a participar, 4 encontravam-se hospitalizados no período, 3 apresentavam-se instáveis hemodinamicamente e 1 não tinha autonomia para decidir sobre sua participação. A maior parte dos indivíduos participantes era do sexo masculino, casados e tinham como nível escolar

Tabela 1. Características da amostra.

\begin{tabular}{|c|c|}
\hline & n (\%) \\
\hline $\begin{array}{l}\text { Sexo } \\
\text { Masculino } \\
\text { Feminino }\end{array}$ & $\begin{array}{l}74(60,7) \\
48(39,3)\end{array}$ \\
\hline $\begin{array}{l}\text { Estado Civil } \\
\text { Solteiro } \\
\text { Casado } \\
\text { Viúvo } \\
\text { Divorciado }\end{array}$ & $\begin{array}{c}11(9,0) \\
79(64,8) \\
20(16,4) \\
12(9,8)\end{array}$ \\
\hline $\begin{array}{l}\text { Escolaridade } \\
\text { Ensino Fundamental Incompleto } \\
\text { Ensino Fundamental Completo } \\
\text { Ensino médio Incompleto } \\
\text { Ensino médio Completo } \\
\text { Superior Incompleto } \\
\text { Superior Completo }\end{array}$ & $\begin{aligned} 77 & (63,1) \\
17 & (13,9) \\
1 & (0,8) \\
20 & (16,4) \\
2 & (1,6) \\
5 & (4,1)\end{aligned}$ \\
\hline $\begin{array}{l}\text { Tabagismo } \\
\text { Sim } \\
\text { Não } \\
\text { Histórico }\end{array}$ & $\begin{array}{c}11(9,0) \\
54(44,3) \\
57(46,7)\end{array}$ \\
\hline $\begin{array}{l}\text { Causa Base } \\
\text { Diabetes Mellitus } \\
\text { Hipertensão Arterial Sistêmica } \\
\text { Glomérulonefrite Crônica } \\
\text { Rins Policísticos } \\
\text { Outras }\end{array}$ & $\begin{array}{c}40(32,8) \\
40(32,8) \\
15(12,3) \\
6(4,9) \\
21(17,2)\end{array}$ \\
\hline $\begin{array}{l}\text { História de Transplante } \\
\text { Sim } \\
\text { Não }\end{array}$ & $\begin{array}{c}12(9,8) \\
110(90,2)\end{array}$ \\
\hline $\begin{array}{l}\text { Membro testado* } \\
\text { Dominante } \\
\text { Não dominante }\end{array}$ & $\begin{array}{c}108(88,5) \\
14(11,5)\end{array}$ \\
\hline $\begin{array}{l}\text { Nível de atividade física } \\
\text { Baixo } \\
\text { Moderado } \\
\text { Alto }\end{array}$ & $\begin{array}{l}67(54,9) \\
43(35,2) \\
12(9,8)\end{array}$ \\
\hline
\end{tabular}


o ensino fundamental incompleto. Mais da metade tinha como diagnóstico causal de IRC diabetes ou hipertensão. A Tabela 1 apresenta as características gerais da amostra.

Os resultados referentes à idade, IMC, tempo de hemodiálise, bioquímica sanguínea, força de preensão manual e nível de atividade física estão demonstrados na Tabela 2. Os indivíduos do sexo masculino apresentaram maior FPM bem como níveis séricos de creatinina e hemoglobina, já os indivíduos do sexo feminino apresentaram, de maneira significativa, melhores índices de KT/V e URR.

Tabela 2. Dados da amostra e comparação das variáveis entre homens e mulheres.

\begin{tabular}{lcccc}
\hline \multicolumn{1}{c}{ Variáveis } & $\begin{array}{c}\text { Amostra } \\
\mathbf{n = 1 2 2}(\mathbf{1 0 0} \%) \\
\text { Média } \pm \mathbf{D P}\end{array}$ & $\begin{array}{c}\text { Homens } \\
\mathbf{n = 7 4}(\mathbf{6 0 , 6} \%) \\
\text { Média } \pm \mathbf{D P}\end{array}$ & $\begin{array}{c}\text { Mulheres } \\
\mathbf{n = 4 8}(\mathbf{3 9 , 4} \%) \\
\text { Média } \pm \mathbf{D P}\end{array}$ & $\boldsymbol{p}$ \\
\hline Idade (anos) & $59,4 \pm 14,0$ & $58,1 \pm 15,2$ & $61,5 \pm 11,8$ & 0,162 \\
IMC (kg/m²) & $24,3 \pm 5,0$ & $24,0 \pm 4,3$ & $24,8 \pm 5,9$ & 0,626 \\
TEMP HD (meses) & $36,0 \pm 43,0$ & $37,0 \pm 46,5$ & $34,4 \pm 37,4$ & 0,929 \\
MET-minutos/semana & $1125,4 \pm 1720,1$ & $1306,5 \pm 1894,8$ & $846,4 \pm 1381,8$ & 0,160 \\
FPM (kgf) & $26,6 \pm 11,6$ & $32,4 \pm 10,1$ & $17,8 \pm 7,3$ & $<0,001^{*}$ \\
Albumina (g/dL) & $3,8 \pm 0,4$ & $3,9 \pm 0,4$ & $3,8 \pm 0,4$ & 0,064 \\
Creatinina (mg/dL) & $10,1 \pm 4,1$ & $10,9 \pm 4,1$ & $8,9 \pm 3,8$ & $0,010^{*}$ \\
KT/V & $1,2 \pm 0,2$ & $1,1 \pm 0,2$ & $1,3 \pm 0,2$ & $<0,001^{*}$ \\
URR (\%) & $63,6 \pm 9,7$ & $60,9 \pm 9,8$ & $67,7 \pm 8,3$ & $<0,001^{*}$ \\
Hematócrito (\%) & $33,5 \pm 6,4$ & $34,0 \pm 6,3$ & $32,8 \pm 6,7$ & 0,290 \\
Hemoglobina (g/dL) & $11,5 \pm 2,6$ & $11,9 \pm 2,7$ & $10,9 \pm 2,3$ & $0,044^{*}$ \\
Colesterol Total (mg/dL) & $144,7 \pm 35,9$ & $138,2 \pm 32,7$ & $154,9 \pm 38,7$ & $0,018^{*}$ \\
Colesterol HDL (mg/dL) & $38,1 \pm 11,8$ & $36,0 \pm 11,0$ & $41,2 \pm 12,5$ & $0,013^{*}$ \\
Colesterol LDL (mg/dL) & $70,9 \pm 39,9$ & $67,5 \pm 29,9$ & $76,4 \pm 39,2$ & 0,294 \\
Triglicerídeos (mg/dL) & $199,3 \pm 103,7$ & $192,7 \pm 106,0$ & $209,3 \pm 100,6$ & 0,240 \\
TFG (ml/min/1,73m²) & $5,8 \pm 3,7$ & $5,8 \pm 3,6$ & $5,9 \pm 3,9$ & 0,669 \\
\hline
\end{tabular}

DP: desvio padrão; IMC: índice de massa corpórea; FPM: força de preensão manual; TEMP HD: tempo de hemodiálise; MET: equivalente metabólico; KT/V: depuração fracional da ureia; URR: taxa de redução da ureia; TFG: taxa de filtração glomerular. $* p<0,05$.

Tabela 3. Comparação das variáveis entre preensão manual abaixo ou igual e acima da média.

\begin{tabular}{lccc}
\hline \multicolumn{1}{c}{ Variáveis } & $\begin{array}{c}\text { FPM } \leq \text { média } \\
\mathbf{n = 6 1}(\mathbf{5 0} \%) \\
\text { Média } \pm \mathbf{D P}\end{array}$ & $\begin{array}{c}\text { FPM }>\text { média } \\
\mathbf{n = 6 1}(\mathbf{5 0} \%)\end{array}$ & $\boldsymbol{p}$ \\
Média $\pm \mathbf{D P}$ & \\
\hline Idade (anos) & $64,2 \pm 12,9$ & $54,7 \pm 13,6$ & $<0,001^{*}$ \\
IMC (kg/m²) & $23,8 \pm 4,6$ & $24,8 \pm 5,4$ & 0,493 \\
Tempo HD (meses) & $40,1 \pm 49,8$ & $31,9 \pm 34,9$ & 0,776 \\
MET-minutos/semana & $809,0 \pm 1432,5$ & $1442,0 \pm 1926,3$ & $0,007^{*}$ \\
Albumina (g/dL) & $3,7 \pm 0,4$ & $3,9 \pm 0,4$ & $0,004^{*}$ \\
Creatinina (mg/dL) & $9,4 \pm 3,9$ & $10,7 \pm 4,2$ & $0,050^{*}$ \\
KT/N & $1,2 \pm 0,2$ & $1,1 \pm 0,2$ & 0,084 \\
URR (\%) & $65,0 \pm 9,3$ & $62,2 \pm 10,1$ & 0,118 \\
Hematócrito $(\%)$ & $32,6 \pm 6,0$ & $34,4 \pm 6,8$ & 0,092 \\
Hemoglobina (g/dL) & $11,0 \pm 2,0$ & $12,0 \pm 3,0$ & $0,025^{*}$ \\
Colesterol Total (mg/dL) & $141,4 \pm 36,0$ & $148,0 \pm 35,9$ & 0,411 \\
Colesterol HDL (mg/dL) & $39,2 \pm 12,3$ & $37,0 \pm 11,4$ & 0,404 \\
Colesterol LDL (mg/dL) & $68,3 \pm 35,7$ & $73,6 \pm 32,1$ & 0,198 \\
Triglicerídeos (mg/dL) & $189,4 \pm 99,7$ & $209,13 \pm 107,7$ & 0,219 \\
TFG (ml/min/1,73m²) & $6,0 \pm 3,2$ & $5,7 \pm 4,2$ & 0,160 \\
\hline
\end{tabular}

DP: desvio padrão; IMC: índice de massa corpórea; FPM: força de preensão manual MET: equivalente metabólico; TEMP HD: tempo de hemodiálise; KT/V: depuração fracional da ureia; URR: taxa de redução da ureia; TFG: taxa de filtração glomerular. $" p<0,05$.
A Tabela 3 apresenta a comparação das variáveis analisadas entre os indivíduos que possuíam FPM $\leq$ média e FPM > média. Foram considerados os valores médios de 32,4 kgf (IC 95\%: 30,1-34,1) para os homens e 17,8 kgf (IC 95\%: 15,6-19,9) para as mulheres. Tiverem melhor desempenho no teste de força os pacientes mais jovens e que referiram praticar mais atividade física, descritos em METs-minutos/semana. Nestes pacientes os níveis séricos de albumina e creatinina encontravam-se mais elevados.

Com relação aos níveis de atividade física baixo e moderado/alto, pacientes que tinham maiores índices de gasto calórico apresentavam melhor desempenho no teste de força e tinham níveis séricos maiores de hemoglobina. Além disso, estes mesmos pacientes possuíam menores valores séricos de colesterol total, se comparado aos pacientes que tinham menores níveis de MET-minutos/semana (Tabela 4).

Utilizando a regressão logística, foi avaliado o odds ratio (OR) das variáveis com diferença significativa em relação à FPM dicotomizada e aos níveis de atividade física baixo e moderado/alto. Observa-se que, na análise da FPM ${ }^{3}$ média, somente a idade e os níveis de albumina mantiveram-se 
Tabela 4. Comparação das variáveis entre o nível de atividade física baixo e moderado/alto.

\begin{tabular}{|c|c|c|c|}
\hline Variáveis & $\begin{array}{c}\text { Nível de atividade física } \\
\text { baixo } \\
\mathrm{n}=67(54,9 \%) \\
\text { Média } \pm \mathrm{DP}\end{array}$ & $\begin{array}{c}\text { Nível de atividade física } \\
\text { moderado/alto } \\
n=55(45,1 \%) \\
\text { Média } \pm \text { DP }\end{array}$ & $p$ \\
\hline Idade (anos) & $60,6 \pm 13,6$ & $57,9 \pm 14,4$ & 0,161 \\
\hline $\mathrm{IMC}\left(\mathrm{kg} / \mathrm{m}^{2}\right)$ & $24,5 \pm 5,9$ & $23,9 \pm 3,6$ & 0,781 \\
\hline Tempo HD (meses) & $39,9 \pm 49,9$ & $31,2 \pm 33,3$ & 0,949 \\
\hline FPM (kgf) & $24,9 \pm 12,6$ & $28,7 \pm 9,8$ & $0,023 *$ \\
\hline Albumina (g/dL) & $3,8 \pm 0,4$ & $3,9 \pm 0,4$ & 0,136 \\
\hline Creatinina $(\mathrm{mg} / \mathrm{dL})$ & $9,7 \pm 3,8$ & $10,5 \pm 4,3$ & 0,383 \\
\hline $\mathrm{KT} / \mathrm{V}$ & $1,2 \pm 0,2$ & $1,1 \pm 0,2$ & 0,463 \\
\hline URR (\%) & $64,8 \pm 8,5$ & $62,1 \pm 10,9$ & 0,235 \\
\hline Hematócrito (\%) & $32,6 \pm 6,2$ & $34,6 \pm 6,5$ & 0,094 \\
\hline Hemoglobina (g/dL) & $10,9 \pm 2,1$ & $12,2 \pm 3,0$ & $0,019 *$ \\
\hline Colesterol Total (mg/dL) & $150,7 \pm 37,2$ & $137,4 \pm 33,2$ & $0,044^{*}$ \\
\hline Colesterol HDL (mg/dL) & $38,9 \pm 11,6$ & $37,1 \pm 12,0$ & 0,298 \\
\hline Colesterol LDL (mg/dL) & $77,1 \pm 37,4$ & $63,3 \pm 27,5$ & 0,058 \\
\hline Triglicerídeos (mg/dL) & $200,4 \pm 99,9$ & $197,8 \pm 109,2$ & 0,705 \\
\hline TFG $\left(\mathrm{ml} / \mathrm{min} / 1,73 \mathrm{~m}^{2}\right)$ & $5,8 \pm 3,2$ & $5,9 \pm 4,2$ & 0,300 \\
\hline
\end{tabular}

DP: desvio padrão; IMC: índice de massa corpórea; FPM: força de preensão manual; TEMP HD: tempo de hemodiálise; KT/V: depuração fracional da ureia; URR: taxa de redução da ureia; TFG: taxa de filtração glomerular. $* p<0,05$.

significativos e, no nível de atividade moderado/alto, apenas os valores de hemoglobina foram significativos (Tabela 5). Esses resultados sugerem que a chance de um indivíduo possuir FPM > média é reduzida 6\% por ano, a partir da média de idade de 59,4 anos e aumenta $253 \%$ por g/dL, em relação á albumina média de $3,8 \mathrm{~g} / \mathrm{dL}$. Ou seja, é muito mais provável que um indivíduo com FPM > média seja mais jovem e possua maiores valores de albumina. Em relação ao nível de atividade física, a chance de um indivíduo possuir

Tabela 5. Odds ratio (OR) das variáveis com diferença significativa em relação à FPM dicotomizada e níveis de atividade física baixo e moderado/alto.

\begin{tabular}{lccc}
\hline \multicolumn{1}{c}{ Variáveis } & \multicolumn{3}{c}{ Regressão Logística } \\
& FPM $>$ média \\
\hline OR & IC 95\% & $\boldsymbol{p}$ \\
Idade (anos) & 0,94 & $0,90-0,97$ & $0,001^{*}$ \\
MET-minutos/semana & 1,00 & $1,00-1,00$ & 0,244 \\
Albumina (g/dL) & 3,53 & $1,10-11,35$ & $0,034^{*}$ \\
Creatinina (mg/dL) & 0,90 & $0,80-1,03$ & 0,134 \\
Hemoglobina (g/dL) & 1,16 & $0,96-1,40$ & 0,123 \\
& \multicolumn{3}{c}{ Regressão Logística } \\
\hline FPM (kgf) & atividade física moderada/alta \\
Hemoglobina $(\mathrm{g} / \mathrm{dL})$ & 1,02 & $0,97-1,05$ & 0,263 \\
Colesterol Total $(\mathrm{mg} / \mathrm{dL})$ & 1,26 & $1,04-1,53$ & $0,038^{*}$ \\
\hline
\end{tabular}

MET: equivalente metabólico; FPM: força de preensão manual. $* p<0,05$. um nível de atividade física moderada/alta aumenta $26 \%$ por $\mathrm{g} / \mathrm{dL}$ em relação a hemoglobina média de $11,5 \mathrm{~g} / \mathrm{dL}$ e diminui $1 \%$ por $\mathrm{mg} / \mathrm{dL}$, quando comparada à média de colesterol total de $144,5 \mathrm{mg} / \mathrm{dL}$. Isto é, indivíduos que possuem nível de atividade física moderada/alta são mais prováveis de estarem com maiores níveis de hemoglobina e menores valores de colesterol total.

\section{DISCUSSÃO}

A amostra do presente estudo demonstrou a superioridade no número de indivíduos do sexo masculino submetidos à HD e a alta prevalência de hipertensão arterial sistêmica (HAS) ou diabetes mellitus (DM) como diagnóstico etiológico da IRC, corroborando o censo de diálise de 2013 no qual $58 \%$ dos pacientes dialisados no Brasil eram homens e o diagnóstico de base dos pacientes em diálise eram, em 65\% das vezes, representados por HAS ou $\mathrm{DM}^{6}$.

Com relação à diferença entre indivíduos do sexo masculino e feminino submetidos a HD, evidenciou-se de maneira significativa que homens possuem maior FPM do que mulheres, bem como evidenciaram maiores níveis plasmáticos de ureia após HD, creatinina e hemoglobina. Em contra partida, mulheres apresentaram valores superiores de Colesterol Total, HDL e KT/V.

Há consenso na literatura sobre homens possuírem maiores níveis de FPM comparado a mulheres em todas as faixas etárias, independente do membro aferido ou da 
ocupação, havendo um declínio nos valores de FPM a partir da 4⿳亠丷厂 década de vida. Na população brasileira, levando-se em conta apenas o membro dominante, a FPM varia de 42,9 a 43,5 kgf em homens e entre 30,5 a 31,7 kgf em mulheres, todos com faixa etária entre 50 e 59 anos $^{22}$. Desta forma, pode-se observar que a amostra do presente estudo apresenta valores abaixo do previsto, demonstrando certo grau de fraqueza muscular.

Valores séricos mais altos de creatinina podem ser explicados por esta ser derivada do metabolismo da creatina muscular, sendo sua produção intimamente relacionada à massa muscular, que é maior em homens, quando comparada a mulheres e maior em jovens, em relação aos idosos ${ }^{23}$. Mulheres apresentaram melhores doses de diálise (KT/V), pois geralmente possuem um menor volume de distribuição da ureia $(\mathrm{V})$, denominador da fórmula que calcula a dose de diálise e que tem como numeradores o valor da depuração de ureia do dialisador $(K)$ e o tempo $(T)^{4,24}$. Comparações adicionais relacionadas a variáveis entre o sexo de pacientes submetidos a HD são limitadas, visto a pequena quantidade de trabalhos confrontando o assunto desta maneira.

Com relação à diferença nos valores de FPM observou-se que indivíduos com maior idade apresentavam FPM abaixo da média. Também foi verificado que a FPM acima da média esteve relacionada com maior nível de atividade física referida, maiores níveis séricos de albumina, creatinina e hemoglobina. Existem evidências na literatura sobre a relação controversa entre albumina sérica e a avaliação nutricional de nefropatas crônicos, porém se sabe que a hipoalbuminemia é um poderoso preditor de mortalidade e que está intimamente ligada a distúrbios adjacentes e a inflamação ${ }^{25,26}$. Nesse sentido, pondera-se a utilização da FPM também como estratificação de risco, pois como demonstrado neste trabalho, é muito mais provável que indivíduos com FPM acima da média possuam maiores valores de albumina.

Leal et al. ${ }^{27}$, ao avaliar 43 pacientes submetidos a HD, encontrou que a FPM em homens foi significativamente maior do que em mulheres, não havendo diferença estatística em fazer o teste antes ou depois da sessão de HD. Concluiu também, diferentemente do presente estudo, que apesar da alta prevalência de diminuição da FPM, este achado não teve relação com variáveis bioquímicas e fatores de diálise como KT/V e URR.

Em outro trabalho, Pedruzzi et al. ${ }^{28}$ encontraram correlação positiva entre FPM, estatura, IMC e alguns dados antropométricos e correlação negativa com idade e eficiência dialítica, corroborando os dados do presente estudo. Os autores acima citados relatam ainda não ter encontrado associação entre hemoglobina e FPM, diferente do presente estudo no qual foi encontrado maior valor de hemoglobina nos indivíduos com maior FPM. No entanto, o resultado obtido corrobora este trabalho, pois, do ponto de vista da fisiopatologia da DRC, sabe-se a deficiência de ferro é um achado frequente ${ }^{29} \mathrm{e}$, consequentemente, níveis de hemoglobina mais baixos podem ser encontrados em pacientes com DRC descompensados.

Alguns estudos sugerem que a FPM possa ser usada como um marcador de comorbidades e para rastreio nutricional na $\mathrm{DRC}^{30,31}$. No entanto, a avaliação do desempenho da extremidade inferior também é indicada como marcador de mortalidade neste perfil de paciente ${ }^{32}$. De qualquer forma, para comparação dos resultados, é importante que se sigam protocolos validados para as diversas avaliações de funcionalidade ${ }^{33}$.

Em se tratando da recomendação e segurança da atividade física em pacientes submetidos à HD, Najas et al. ${ }^{34}$, em uma revisão de 12 trabalhos comparando a eficiência entre a prática de treinamento físico no período interdialítico e intradialítico, concluíram que ambos os programas eram seguros e efetivos, sendo que se tratavam de propostas não farmacológicas, com efeitos incrementais em vários parâmetros relacionados ao paciente e à qualidade da diálise.

No que se refere ao nível de atividade física utilizado para classificar indivíduos entre sedentários e não sedentários, existem certas controvérsia ${ }^{16}$. As recomendações utilizadas pelo IPAQ, em suma, consideram suficientemente ativos indivíduos que atinjam 600 MET-minuto por semana, com qualquer combinação de exercício moderado ou vigoroso ${ }^{19}$. No Brasil, são adotados os critérios propostos pela OMS que orienta a prática de pelo menos 150 minutos de atividade física moderada ou 75 minutos de atividade física rigorosa por semana em sessões de pelo menos 10 minutos $^{35}$. Desta forma, percebe-se que a amostra do presente estudo demonstrou, no geral, menores níveis de atividade física, apontando razoável sedentarismo nestes pacientes.

Neste trabalho, indivíduos com atividade física referida moderada/alta tiveram relação direta com maiores níveis de Hemoglobina e inversa com níveis séricos de colesterol total. Dados semelhantes em relação aos maiores níveis de hemoglobina relacionado ao exercício físico foram encontrados no trabalho de Henrique et al. ${ }^{36}$, no qual 14 pacientes foram analisados antes e após 12 semanas de treinamento aeróbico intradialítico. Apesar do achado concordante, o estudo também evidenciou maiores níveis de colesterol LDL ao final das 12 semanas, indo contra ao que foi apresentado neste estudo. Em outro trabalho, Ribeiro et al. ${ }^{37}$, ao avaliarem 60 pacientes em HD, diabéticos e não diabéticos submetidos ao exercício resistido por 8 semanas, encontrou significativa melhora na qualidade de vida dos 
pacientes participantes, mesmo não evidenciando melhora nos parâmetros bioquímicos.

Levando-se em conta que a principal causa de morte de pacientes em HD são as doenças cardiovasculares ${ }^{2}$ e que a relação entre o perfil lipídico e exercícios aeróbicos são bem definidos ${ }^{38}$, torna-se mandatória o incentivo de atividades aeróbicas ou de resistência ${ }^{2}$ pelos médicos assistentes e pela equipe multidisciplinar que acompanha. Apesar dos dados discordantes na literatura sobre atividade física, FPM a dados laboratoriais ${ }^{31}$, existem trabalhos documentando que níveis de atividade física estão associados a menos sintomas depressivos e a maiores níveis cognitivos por parte do paciente ${ }^{39,40}$.

Em relação às limitações do estudo, pode-se comentar que existem algumas variações entre protocolos posturais utilizados na mensuração da FPM. Além disso, o possível viés da avaliação do nível de atividade física por meio do questionário IPAQ que, apesar de ser validado, possui aspectos subjetivos que podem influenciar na análise. E o delineamento transversal do estudo, que limita outras comparações e causalidade.

Em síntese, pode-se concluir que a FPM acima da média foi encontrada principalmente em pacientes mais jovens, que referiram praticar mais atividade física e possuíam maiores níveis séricos de albumina e creatinina. Em relação ao moderado/alto nível de atividade física, foi observada maior FPM, maiores níveis de hemoglobina e menores valores de colesterol total. Os maiores valores de creatinina podem ser metabolicamente relacionados com o sexo masculino e maior massa muscular. Além disso, a amostra do presente estudo apresentou maior fraqueza muscular quando comparado com a mesma faixa etária em indivíduos saudáveis e uma predominância de baixo nível de atividade física, ou seja, sedentarismo. Conclui-se que o maior nível de atividade física e maior FPM, de forma geral, estão relacionados a aspectos positivos em pacientes renais crônicos, aventando a proposta de albumina e hemoglobina como marcadores do estado da DRC.

\section{REFERÊNCIAS}

1. Romão Jr JE: Diretrizes Brasileiras da Doença Renal Crônica. Doença Renal Crônica: definição, epidemiologia e classificação. J Bras Nefrol. 2004;26(3):4-5.

2. Nascimento LCA, Coutinho EB, Silva KNG. Efetividade do Exercício Físico na Insuficiência Renal Crônica. Fisioter Mov. 2012;25(1):2319. https://doi.org/10.1590/S0103-51502012000100022

3. Silva MMH, Brune MFSS. Importância do cálculo da taxa de filtração glomerular na avaliação da função renal de adultos. Rev Bras Farm. 2011;92(3):160-5.

4. Breitsameter G, Figueiredo AE, Kochhann DS. Cálculo de Kt/V em hemodiálise: comparação entre fórmulas. J Bras Nefrol. 2012; $34(1): 22-6$
5. Longo, DL, Braunwald E, Kasper DL, Fauci AS, Jameson JR, Hauser S. Medicina interna de Harrison. 18 $8^{\underline{a}}$ ed. Porto Alegre: $\mathrm{AMGH}$; 2013

6. Sociedade Brasileira de Nefrologia: Censo de Diálise [Internet] São Paulo (SP): Sociedade Brasileira de Nefrologia; 2013 [cited 2014 Abr]. Available from: http://sbn.org.br/pdf/censo 2013 publico leigo.pdf

7. Peres LAB, Biela R, Herrmann M, Matsuo T, Ann HK, Camargo MTA, Rohde NRS, Uscocovich VSM. Estudo epidemiológico da doença renal crônica terminal no oeste do Paraná: uma experiência de 878 casos atendidos em 25 anos. J Bras Nefrol. 2010;32(1):51-6. https:// doi.org/10.1590/S0101-28002010000100010

8. Melo FGC. Análise de Sobrevida de Pacientes Renais Crônicos em Hemodiálise [dissertação]. Fortaleza: Universidade Federal do Ceará; 2006. $123 \mathrm{f}$.

9. Sesso R; Centro de Vigilância Epidemiológica da Secretaria de Estado da Saúde de São Paulo. Epidemiologia da doença renal crônica no Brasil e sua prevenção. São Paulo; 2007.

10. Frazão CMFQ, Ramos VP, Lira ALBC. Qualidade de vida de pacientes submetidos à hemodiálise. Rev Enferm UERJ. 2011;19(4): 577-82.

11. Medeiros RH, Pinent DED, Meyer F. Aptidão física de indivíduo com doença renal crônica. J Bras Nefrol. 2002;24(2):81-7.

12. Dias JA, Ovando AC, Külkamp W, Borges, NG Jr. Força de preensão palmar: métodos de avaliação e fatores que influenciam na medida. Rev Bras Cineantropom Desempenho Hum. 2010;12(3):209-16.

13. Roberts HC, Denison HJ, Martin HJ, Patel HP, SYDDALL H, Cooper C, Sayer AA. A review of the measurement of grip strength in clinical and epidemiological studies: towards a standardised approach. Age Ageing. 2011;40(4):423-9. https://doi.org/10.1093/ageing/afr051

14. National Kidney Foundation: K/DOQI clinical practice guidelines for cardiovascular disease in dialysis patients. Am J Kidney Dis. 2005;45[Suppl 3]:S1-S154.

15. Johansen KL. Exercise in the end-stage renal disease population. J Am Soc Nephrol. 2007;18:1845-54. https://doi.org/10.1681/ ASN.2007010009

16. Lima DF, Levy RB, Luiz OC. Recomendação para atividade física e saúde: consensos, controvérsias e ambiguidades. Rev Panam Salud Publica. 2014;36(3):164-70.

17. Chang YT, Wu HL, Guo HR, Cheng YY, Tseng CC, Wang MC, Lin CY, Sung JM. Handgrip strength is an independent predictor of renal outcomes in patients with chronic kidney diseases. Nephrol Dial Transplant. 2011;26(11):3588-95. https://doi.org/10.1093/ndt/ gro13

18. Vespasiano BS, Dias R, Correa DA. A utilização do Questionário Internacional de Atividade Física (Ipaq) como ferramenta diagnóstica do nível de aptidão física: uma revisão no Brasil. Saúde Rev. 2012;12(32):49-54. https://doi.org/10.15600/2238-1244/ sr.v12n32p49-54

19. International Physical Activity Questionnaire (SE). Guidelines for Data Processing and Analysis of the International Physical Activity Questionnaire. Suécia: International Physical Activity Questionnaire; 2005.

20. American Society of Hand Therapists. Clinical assessment recommendations. Chicago; 1992. 
21. Dias JA, Ovando AC, Külkamp W, Junior NGB. Força de preensão palmar: métodos de avaliação e fatores que influenciam a medida. Rev Bras Cineantropom Desempenho Hum. 2010;12(3):209-16.

22. Nascimento MF, Benassi R, Caboclo FD, Salvador ACS, Gonçalves LCO. Valores de referencia de força de preensão manual em ambos os gêneros e diferentes grupos etários. Um estudo de revisão [Internet]. Buenos Aires: EFDeportes.com, Revista Digital; [uptodate 2010 Dez; cited 2015 Maio]. Available from: http://www.efdeportes. com/efd151/forca-de-preensao-manual-em-ambos-os-generos.htm

23. Bastos MG. Biomarcadores na nefrologia [Internet]. E-book. São Paulo: Sociedade Brasileira de Nefrologia; 2011 [uptodate 2010 Dez; cited 2015 Maio]. Available from: http://www.sbn.org.br/pdf/ biomarcadores.pdf

24. Daugirdas JT, Ing TS. Prescrição de hemodiálise crônica: uma abordagem da cinética da uréia. In: Daugirdas JT, Ing TS. Manual de diálise. 3를 ed. Rio de Janeiro: Medsi; 2003. Cap. 9.

25. Friedman AN, Fadem SZ. Reassessment of albumin as a nutritional marker in kidney disease. J Am Soc Nephrol. 2010;21:223-30. https://doi.org/10.1681/ASN.2009020213

26. Gupta J, Mitra N, Kanetsky PA, Devany J, Wing MR, Reilly M, Shah VO, Balakrishnan VS, Guzman NJ, Girndt M, Periera BG, Feldman HI, Kusek JW, Joffe MM, Raj DS. Association between Albuminuria, Kidney Function, and Inflammatory Biomarker Profile in CKD in CRIC. Clin J Am Soc Nephrol. 2012;7:1938-46. https://doi. org/10.2215/CJN.03500412

27. Leal VO, Stockler-Pinto MB, Farage NE, Aranha LN, Fouque D, Anjos LA, Mafra D. Handgrip strenght and its dialysis determinants in hemodialysis patients. Nutrition. 2011;27(11):1125-9. https:// doi.org/10.1016/j.nut.2010.12.012

28. Pedruzzi LM, Leal VO, Barros AF, Lobo JC, Mafra D. Fatores relacionados à força de preensão manual de pacientes submetidos à hemodiálise: ênfase na anemia. Nutrire. 2012;37(1):22-33. https:// doi.org/10.4322/nutrire.2012.003

29. Abensur H. Deficiência de ferro na doença renal crônica. Rev Bras Hematol Hemoter. 2010;32(Suppl 2):95-8. https://doi.org/10.1590/ S1516-84842010005000047

30. Dantas MAS. Investigação de associações entre força de preensão manual medida por dinamômetro com estado nutricional e a sobrevida de pacientes iniciando hemodiálise de manutenção: estudo PROHEMO. [monografia]. Salvador (BA): Universidade Federal da Bahia; 2014.
31. Leal VO, Mafra D, Fouque D, Anjos LA. Use of handgrip strength in the assessment of the muscle function of chronic kidney disease patients on dialysis: a systematic review. Nephrol Dial Transplant. 2011;26:1354-60. https://doi.org/10.1093/ndt/gfq487

32. Roshanravan B, Robinson-Cohen C, Patel KV, Ayers E, Littman AJ, Boer IH, Ikizler TA, Himmelfarb J, Katzel LI, Kestenbaum B, Seliger S. Association between physical performance and all-cause mortality in CKD. J Am Soc Nephrol. 2013;24:822-30. https://doi.org/10.1681/ ASN.2012070702

33. Schulüssel MM, Anjos LA, Kac G. A dinamometria manual e seu uso na avaliação nutricional. Rev Nutr Campinas. 2008;21(2):223-35. https://doi.org/10.1590/s1415-52732008000200009

34. Najas CS, Pissulin FDM, Pacagnelli FL, Betônico GN, Almeida IC, Neder JA. ᄀSegurança e eficácia do treinamento físico na insuficiência renal crônica. Rev Bras Med Esporte. 2009;15(5): 384-8. https://doi.org/10.1590/S1517-86922009000600013

35. World Health Organization. Global recommendations on physical activity for health [Internet]. Genebra: WHO; 2010 [cited 2015 Maio]. Available from: http://whqlibdoc.who.int/ publications/2010/9789241599979_eng.pdf

36. Henrique DMN, Reboredo MM, Chaoubah A, Paula RB. Treinamento aeróbico melhora a capacidade funcional de pacientes em hemodiálise crônica. Arq Bras Cardiol. 2010;94(6):823-8. https://doi.org/10.1590/S0066-782X2010005000043

37. Ribeiro R, Coutinho GL, Iuras A, Barbosa AM, Souza JAC, Diniz DP, Schor N. Efeito do exercício resistido intradialítico em pacientes renais crônicos em hemodiálise. J Bras Nefrol. 2013;35(1):13-9. https://doi.org/10.5935/01012800.20130003

38. Prado ES, Dantas EHM. Efeitos dos exercícios físicos aeróbio e de força nas lipoproteínas HDL, LDL e lipoproteína (a). Arq Bras Cardiol. 2002;79(4):429-33. https://doi.org/10.1590/S0066-782X 2002001300013

39. Stringuetta-Belik F, Shiraishi FG, Silva VRO, Barretti P, Caramori JCT, Bôas PJF, Martin LC, Franco RJS. Maior nível de atividade física associa-se a melhor função cognitiva em renais crônicos em hemodiálise. J Bras Nefrol. 2012;34(4):378-86. https://doi. org/10.5935/0101-2800.20120028

40. Cavalcanti CTA, Araújo JC Filho, Marinho PEM. Nível de atividade física e sintomas depressivos em pacientes submetidos à hemodiálise: um estudo de corte transversal. Fisioter Pesq. 2014;21(2):161-6. https://doi.org/10.1590/1809-2950/49921022014 